\title{
CRITICAL CROSS POWER SPECTRAL DENSITY FUNCTIONS AND THE HIGHEST RESPONSE OF MULTI-SUPPORTED STRUCTURES SUBJECTED TO MULTI-COMPONENT EARTHQUAKE EXCITATIONS
}

\author{
ABHIJIT SARKAR* AND C. S. MANOHAR ${ }^{\dagger}$ \\ Department of Civil Engineering, Indian Institute of Science, Bangalore 560012, India
}

\begin{abstract}
SUMMARY
The highest response of multi-supported structures subjected to partially specified multi-component earthquake support motions is considered. The seismic inputs are modelled as incompletely specified vector Gaussian random processes with known autospectral density functions but unknown cross spectral densities and these unknown functions are determined such that the steady state response variance of a given linear system is maximized. The resulting cross power spectral density functions are shown to be dependent on the system properties, autospectra of excitation and the response variable chosen for maximization. It emerges that the highest system response is associated neither with fully correlated support motions, nor with independent motions, but, instead, specific forms of cross power spectral density functions are shown to exist which produce bounds on the response of a given structure. Application of the proposed results is demonstrated by examples on a ground based extended structure, namely, a $1578 \mathrm{~m}$ long, three span, suspension cable bridge and a secondary system, namely, an idealized piping structure of a nuclear power plant.
\end{abstract}

KEY WORDS: critical seismic excitations; multi-support seismic excitations

\section{INTRODUCTION}

Spatially extended structures such as long span bridges and pipelines and internal secondary structures such as piping in nuclear power plants are multi-supported and each support is subjected to multi-component support motions during an earthquake. Design earthquake support motions for these structures can be specified in terms of time histories, response spectra or random processes/fields. For single point excitations, the relationship between these three forms of seismic load specifications is well understood. ${ }^{1}$ On the other hand, for multi-support and multi-component excitations, these relationships, especially those between response spectra and random field models, are not obvious. Moreover, the accuracy and robustness of these seismic input models are open to question in view of the significant uncertainties which are present in the earthquake excitation mechanism on the one hand, and the scarcity of recorded strong motion data on the other. This is more so, especially when dealing with multi-supported structures subjected to multi-component excitations, for which, in addition to the power spectra or response spectra at every support point, the information on cross correlations between components of excitation at each of the supports and phase lag and coherency loss effects due to seismic wave transmission between supports also need to be specified in terms of the cross power spectral density functions, the data needed for which are all the more scarce. Given this ill-posed nature of the problem, it is useful to ask as to what is the worst that might happen to a given structure under a set of incompletely specified seismic loading conditions. The method of critical excitations has been developed to answer this type of question: these excitations are defined as those which produce the

\footnotetext{
*Research Student

${ }^{\dagger}$ Assistant Professor

CCC 0098-8847/96/030303-13

(C) 1996 by John Wiley \& Sons, Ltd.
}

Received 20 June 1995 Revised 17 November 1995 
highest damage to a given structure and at the same time satisfy a set of constraints reflecting some of the well known features of earthquake time histories. Thus, the critical excitations, by definition, depend upon the system under consideration, nature of partial information available and the damage variable chosen for optimization and their determination constitutes an inverse problem in structural dynamics. Some representative works which may be cited in this context are those by Drenick ${ }^{2,3}$ on critical time histories, by Wang and $\mathrm{Yun}^{4}$ and Srinivasan et al. ${ }^{5}$ on critical response spectra, by Iyengar and Manohar ${ }^{6}$ and Manohar and Sarkar ${ }^{7}$ on critical random process models and by Sarkar and Manohar ${ }^{8}$ on critical vector random excitations. A review of relevant literature on this topic has been recently presented by Manohar and Sarkar. $^{7}$

The present authors are currently involved in the development of critical random process and random field models for earthquake loads on single support and multi-support structures. ${ }^{78}$ In these studies, the seismic input is taken to be Gaussian distributed with specified total average energy, zero crossing rate and frequency content and an unknown Power Spectral Density (PSD) function/matrix. The unspecified parameters of the input process are determined such that the response variance of a given linear structure is maximized. Some important conclusions which have emerged so far from these studies are the following:

(1) Critical excitations as defined above have a nearly deterministic structure and are strongly resonant in character. This unrealistic nature constitutes a shortcoming in the definition of critical excitation. This can be overcome by incorporating a quantitative measure of uncertainty in terms of the entropy rate of the excitation process in the definition of critical excitation. ${ }^{7}$

(2) The problem of critical excitation for single point excitation is linear in nature and, therefore, can be solved within the framework of linear programming methods. For multi-support critical excitation, however, the optimization problem becomes non-linear in nature. ${ }^{8}$

(3) For structures with two supports, the highest response is produced by excitations which have specific forms of partial correlations and not by excitations which are fully correlated or fully uncorrelated.

In this paper, we extend the above studies to construct critical seismic excitation models including the effects of multi-component support excitations on multi-supported structures. The seismic excitation of each support is taken to be a stationary vector Gaussian random process consisting of translation components. We focus our attention on the situation in which a prior knowledge of the autospectral density function of these excitations is available but the cross correlation properties are not known. The aim of the study is to determine the critical coherence functions for components of excitations at a given support and also for components of excitations at two distinct supports, which maximize the steady state response variance of a given structure.

As has been noted already, in the context of earthquake engineering, multi-support structures occur in two different situations: firstly, in ground based extended structures whose principal dimensions are longer than the characteristic seismic wavelength, and, therefore, effects of coherency loss and phase lag need to be accounted for in the models for the seismic inputs, and, secondly, secondary systems which are supported at different floor levels in an industrial building. The seismic analysis of extended primary structures is facilitated by random field models which allow for spatial variability of earthquake ground motions. The construction of these models has received impetus after the recent availability of dense seismic array data and several studies in the recent past have been conducted on seismic response analysis of extended structures $^{9-12}$; see Reference 13 for a comprehensive review on this topic. Similarly, for multi-supported secondary structures, both response spectrum and power spectrum based approaches have been employed; see, for example, References 14-18. Furthermore, characterization of earthquake ground motions as being multi-component in nature has also received considerable attention; see, for example, Reference 19. A distinguishing feature of these problems has been the need to model the cross correlation between the different excitation components. It is through this function that the coherency loss and phase lag effects of multisupport excitations get reflected. The study reported in the present paper offers an alternative approach to the modelling of the Cross Power Spectral Density (CPSD) functions and it will be demonstrated that highest 


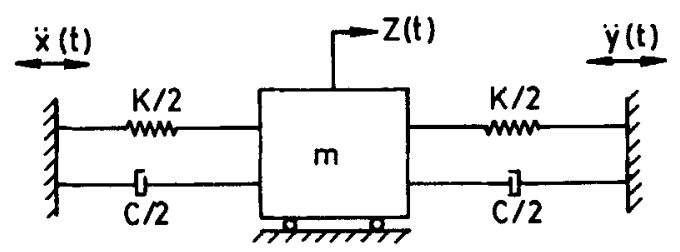

Figure 1. SDOF system with double support excitations

responses occur when he CPSD functions have specific forms which neither correspond to fully correlated motions nor to uncorrelated motions, but, instead, are dependent on the property of the structure under consideration. Illustrative examples on a $1578 \mathrm{~m}$ three span suspended cable structure and an idealized piping structure of a nuclear power plant are presented which bring out different features of the proposed solutions.

\section{SINGLE DEGREE OF FREEDOM SYSTEMS}

Figure 1 shows a SDOF system supported at two points and subjected to support accelerations $\ddot{x}$ and $\ddot{y}$ which are modelled as a pair of stationary Gaussian band-limited random processes with the PSD matrix given by

$$
S(\omega)=\left(\begin{array}{ll}
S_{x x}(\omega) & S_{x y}(\omega) \\
S_{y x}(\omega) & S_{y y}(\omega)
\end{array}\right)
$$

Furthermore, in this study we take cross spectral density to be of the form

$$
S_{x y}(\omega)=\left|S_{x y}(\omega)\right| \exp [-\mathrm{i} \omega \tau]
$$

where $\tau=L / V$ is the seismic transmission time lag over a span $L$ with an apparent velocity of propagation $V$. The governing equation of motion for the total displacement $z_{t}(t)$ is given by

$$
m \ddot{z}_{\mathrm{t}}+c\left\{\dot{z}_{\mathrm{t}}-\frac{\dot{x}+\dot{y}}{2}\right\}+k\left\{z_{\mathrm{t}}-\frac{x+y}{2}\right\}=0
$$

In terms of the dynamic displacement $z=z_{\mathbf{t}}-\frac{1}{2}(x+y)$, one gets

$$
\ddot{z}+2 \eta \omega_{\mathrm{n}} \dot{z}+\omega_{\mathrm{n}}^{2} z=-\frac{1}{2}(\ddot{x}+\ddot{y})
$$

Let us consider the force $F$ in the left spring as the response variable of interest. The steady state variance of the quantity $4 F / k$ can be shown to be given by

$$
\sigma^{2}=\int_{\omega_{1}}^{\omega_{2}}\left\{S_{x x}(\omega) H_{1}(\omega)+S_{y y}(\omega) H_{2}(\omega)+\left|S_{x y}(\omega)\right| H_{12}(\omega)\right\} \mathrm{d} \omega
$$

Here $\left(\omega_{1}, \omega_{2}\right)$ is the frequency bandwidth and the generalized transfer functions $H_{1}, H_{2}$ and $H_{12}$ are given by

$$
\begin{gathered}
H_{1}(\omega)=\left\{\frac{1}{\omega^{4}}+\frac{1}{\left(\omega^{2}-\omega_{0}^{2}\right)^{2}+\left(2 \eta \omega \omega_{0}\right)^{2}}+\frac{2\left(\omega^{2}-\omega_{0}^{2}\right)}{\omega^{2}\left[\left(\omega^{2}-\omega_{0}^{2}\right)^{2}+\left(2 \eta \omega \omega_{0}\right)^{2}\right]}\right\}\left|H_{\mathrm{f}}(\omega)\right|^{2} \\
H_{2}(\omega)=\left\{\frac{1}{\omega^{4}}+\frac{1}{\left(\omega^{2}-\omega_{0}^{2}\right)^{2}+\left(2 \eta \omega \omega_{0}\right)^{2}}-\frac{2\left(\omega^{2}-\omega_{0}^{2}\right)}{\omega^{2}\left[\left(\omega^{2}-\omega_{0}^{2}\right)^{2}+\left(2 \eta \omega \omega_{0}\right)^{2}\right]}\right\}\left|H_{\mathrm{f}}(\omega)\right|^{2} \\
H_{12}(\omega)=\left\{\frac{-2 \cos \omega \tau}{\omega^{4}}+\frac{2 \cos \omega \tau}{\left(\omega^{2}-\omega_{0}^{2}\right)^{2}+\left(2 \eta \omega \omega_{0}\right)^{2}}+\frac{8 \eta \omega \omega_{0} \sin \omega \tau}{\omega^{2}\left[\left(\omega^{2}-\omega_{0}^{2}\right)^{2}+\left(2 \eta \omega \omega_{0}\right)^{2}\right]}\right\}\left|H_{f}(\omega)\right|^{2}
\end{gathered}
$$


The filter function $H_{\mathrm{f}}(\omega)$ appearing in these equations is given by

$$
H_{\mathrm{f}}(\omega)=\frac{\left(\omega / \omega_{\mathrm{f}}\right)^{4}}{\left[1-\left(\omega / \omega_{\mathrm{f}}\right)^{2}\right]^{2}+\left[4 \zeta_{\mathrm{f}}^{2}\left(\omega / \omega_{\mathrm{f}}\right)^{2}\right]}
$$

this being an artefact to remove the singularity in the support displacements at $\omega=0$ which would otherwise be present. ${ }^{20}$ It is assumed in this study that $\omega_{\mathrm{f}}=5.5 \mathrm{rad} / \mathrm{s}$ and $\zeta_{\mathrm{f}}=0.53$.

Notice that each of the functions in equations (6)-(8), $H_{1}, H_{2}$ and $H_{12}$, is expressed as a sum of three terms, and, accordingly, the response as given by equation (5) can also be written in the form

$$
\sigma^{2}=\sigma_{\mathrm{ps}}^{2}+\sigma_{\mathrm{d}}^{2}+\sigma_{\mathrm{c}}^{2}
$$

where

$$
\begin{gathered}
\sigma_{\mathrm{ps}}^{2}=\int_{\omega_{1}}^{\omega_{2}}\left\{\frac{1}{\omega^{4}}\left[S_{x x}(\omega)+S_{y y}(\omega)-2 \cos \omega \tau\left|S_{x y}(\omega)\right|\right]\right\}\left|H_{\mathrm{f}}(\omega)\right|^{2} \mathrm{~d} \omega \\
\sigma_{\mathrm{d}}^{2}=\int_{\omega_{1}}^{\omega_{2}}\left\{\frac{1}{\left(\omega^{2}-\omega_{0}^{2}\right)^{2}+\left(2 \eta \omega \omega_{0}\right)^{2}}\left[S_{x x}(\omega)+S_{y y}(\omega)+2 \cos \omega \tau\left|S_{x y}(\omega)\right|\right]\right\}\left|H_{\mathrm{f}}(\omega)\right|^{2} \mathrm{~d} \omega \\
\sigma_{\mathrm{c}}^{2}=\int_{\omega_{1}}^{\omega_{2}} \frac{1}{\omega^{2}\left[\left(\omega^{2}-\omega_{0}^{2}\right)^{2}+\left(2 \eta \omega \omega_{0}\right)^{2}\right]}\left[2\left(\omega^{2}-\omega_{0}^{2}\right)\left(S_{x x}-S_{y y}\right)+8 \eta \omega \omega_{0} \sin \omega \tau\left|S_{x y}\right|\right]\left|H_{\mathrm{f}}\right|^{2} \mathrm{~d} \omega
\end{gathered}
$$

These three response components represent, respectively, the pseudo-static part, the dynamic part and the term arising from the correlation between pseudo-static and dynamic response components. This is in contrast with the representation given by equation (5), in which the response variance again consists of three components which are, respectively, the contributions from $\ddot{x}, \ddot{y}$ and from correlations between $\ddot{x}$ and $\ddot{y}$. The form given in equation (10) is consistent with that reported in literature, ${ }^{18}$ but, as will be shown later, for the purpose of this paper, the alternative form given in equation (5) proves to be more useful.

In formulating the problem of critical excitations we assume that the knowledge of $S_{x x}(\omega)$ and $S_{y y}(\omega)$ is available and it is required to find $\left|S_{x y}(\omega)\right|$ which produces the highest response variance under the constraint

$$
0 \leqslant\left|S_{x y}(\omega)\right| \leqslant \sqrt{S_{x x}(\omega) S_{y y}(\omega)}
$$

Notice that the objective function in this optimization problem, vide equation (5), is a linear function of $\left|S_{x y}(\omega)\right|$ and so is the constraint as given above. Consequently, one can use linear programming methods to achieve the solutions. However, a much simpler alternative is possible. In order to see this, we begin by noting that the functions $H_{1}$ and $H_{2}$ as given by equations ( 6 and 7 ) can also be written in the form

$$
H_{1}(\omega)=\frac{\left(2 \omega^{2}-\omega_{0}^{2}\right)^{2}+\left(2 \eta \omega \omega_{0}\right)^{2}}{\omega^{4}\left[\left(\omega^{2}-\omega_{0}^{2}\right)^{2}+\left(2 \eta \omega \omega_{0}\right)^{2}\right]}\left|H_{\mathrm{f}}(\omega)\right|^{2}
$$

and

$$
H_{2}(\omega)=\frac{\omega_{0}^{2}\left(\omega_{0}^{2}+4 \eta^{2} \omega^{2}\right)}{\omega^{4}\left[\left(\omega^{2}-\omega_{0}^{2}\right)^{2}+\left(2 \eta \omega \omega_{0}\right)^{2}\right]}\left|H_{\mathrm{f}}(\omega)\right|^{2}
$$

from which it follows that the functions $H_{1}(\omega)$ and $H_{2}(\omega)$ are positive. The function $H_{12}(\omega)$, as can be seen from equation (8), can, however, take both positive and negative values. Consequently, the contributions made by the first two terms in the integrand in equation (5) are always positive, while, on the other hand, the 
last term can make either positive or negative contributions. From this, it follows that the critical $\left|S_{x y}(\omega)\right|$ which produces the highest variance is given by

$$
\begin{gathered}
\left|S_{x y}(\omega)\right|=0 \quad \text { if } H_{12}(\omega, \tau)<0 \\
\left|S_{x y}(\omega)\right|=\sqrt{S_{x x}(\omega) S_{y y}(\omega)} \text { if } H_{12}(\omega, \tau)>0
\end{gathered}
$$

Thus, the critical cross PSD depends on the transfer function $H_{12}(\omega, \tau), S_{x x}(\omega)$ and $S_{y y}(\omega)$ and the highest response occurs neither when $\ddot{x}(t)$ and $\ddot{y}(t)$ are fully coherent nor when they are fully incoherent, but, instead, when the two processes have the specific correlation properties as given above. Conversely, the most favourable $\left|S_{x y}(\omega)\right|$ which results in the least response variance can be easily seen to be of the form

$$
\begin{gathered}
\left|S_{x y}^{*}(\omega)\right|=0 \quad \text { if } H_{12}(\omega, \tau)>0 \\
\left|S_{x y}^{*}(\omega)\right|=\sqrt{S_{x x}(\omega) S_{y y}(\omega)} \quad \text { if } H_{12}(\omega, \tau)<0
\end{gathered}
$$

Thus, the response variance produced by any other CPSD function will lie between those produced by $\left\{S_{x y}(\omega) \mid\right.$ and $\left|S_{x y}^{*}(\omega)\right|$. For the purpose of illustration, consider a SDOF system with $\omega_{0}=20 \cdot 0 \mathrm{rad} / \mathrm{s}$ and $\eta=0.05$ which is acted upon by $\ddot{x}$ and $\ddot{y}$ having identical autospectral density functions given by

$$
S_{0}(\omega)=\frac{\phi_{0}\left[1+4 \zeta_{\mathrm{g}}^{2}\left(\omega / \omega_{\mathrm{g}}\right)^{2}\right]}{\left[1-\left(\omega / \omega_{\mathrm{g}}\right)^{2}\right]^{2}+4 \zeta_{\mathrm{g}}^{2}\left(\omega / \omega_{\mathrm{g}}\right)^{2}}
$$

with $\zeta_{\mathrm{g}}=0.6$ and $\omega_{\mathrm{g}}=15.0 \mathrm{rad} / \mathrm{s}$. The time lag is taken to be $\tau=1 \mathrm{~s}$. The PSD of the quantity $4 F / k$ is shown in Figure 2(a) for four excitation cases, namely, when $\ddot{x}$ and $\ddot{y}$ are independent, when they are fully coherent, when $\left|S_{x y}\right|$ is critical and when it is most favourable. It must be noted that the multiple peaks observed in these plots arise due to the effect of time lag $\tau$. The variances of the quantity for these four cases, normalized with respect to $\phi_{0}$, are found to be, respectively, 0.023, 0.025, 0.030 and 0.017. Clearly, the critical and the most favourable CPSD functions produce, respectively, the upper and lower bounds on the response. This can also be seen in Figure 2(b), in which the response as a function of $\tau$ is shown for the four excitation cases mentioned above. For large time lags, it is obvious that the results for the cases of fully coherent and independent excitations lead to the same response levels. On the other hand, for small $\tau$, however, the responses produced by fully coherent excitations are observed to fluctuate about the response level due to independent excitations. It can be observed from Figure 2(a) that the critical response PSD function at any frequency equals the higher of the two PSD functions which correspond to the cases when $\ddot{x}$ and $\ddot{y}$ are independent and when they are fully correlated. This provides the insight into the mechanism by which the highest response is produced. The situation would become somewhat complicated when more than two excitation components are present in the problem as in the case of multi-component excitation of multisupported structures. The next two sections consider two examples which illustrate this.

\section{THREE SPAN SUSPENDED CABLE}

Figure 3 shows a three span suspended cable structure with each of the four supports acted upon by a two dimensional earthquake support motion. In the numerical work, it is assumed that $L_{1}=288.6 \mathrm{~m}$, $L_{2}=1000 \mathrm{~m}, \theta=30^{\circ}$, Young's modulus $E=8.75 \times 10^{10} \mathrm{~N} / \mathrm{m}^{2}$, area of cross section $A=9.62 \times 10^{-4} \mathrm{~m}^{2}$, mass per unit length $m=23.6 \mathrm{~kg} / \mathrm{m}$, cable tension $H=32837.0 \mathrm{~N}$ and viscous damping $C=0.2 \mathrm{Ns} / \mathrm{m}^{2}$. The autospectral density functions, $S_{i i}(\omega), i=1, \ldots, 8$, of the individual components are again taken to be of the form given by equation (19) with $\omega_{\mathrm{g}}=15.0 \mathrm{rad} / \mathrm{s}$ and $\zeta_{\mathrm{g}}=0.6$. The unspecified cross spectral density functions are taken to be of the form given by equation (2). The problem is to find these unspecified functions which would maximize the steady state variance of the system response. For the purpose of illustration we consider two response variables, namely, the forces transmitted to the support along the directions 3 and 4.

The first step in solving this problem is to derive the input-output relations and for this purpose we follow the direct dynamic stiffness approach. ${ }^{21}$ This consists of first computing the element dynamic stiffness 


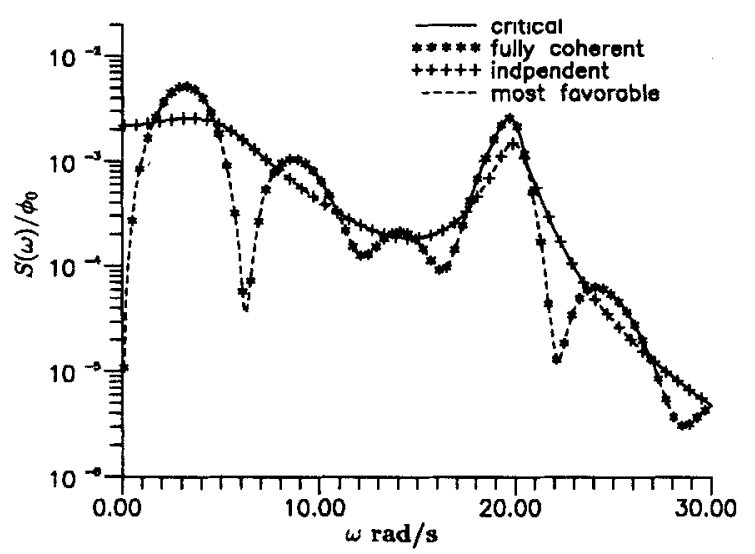

(a)

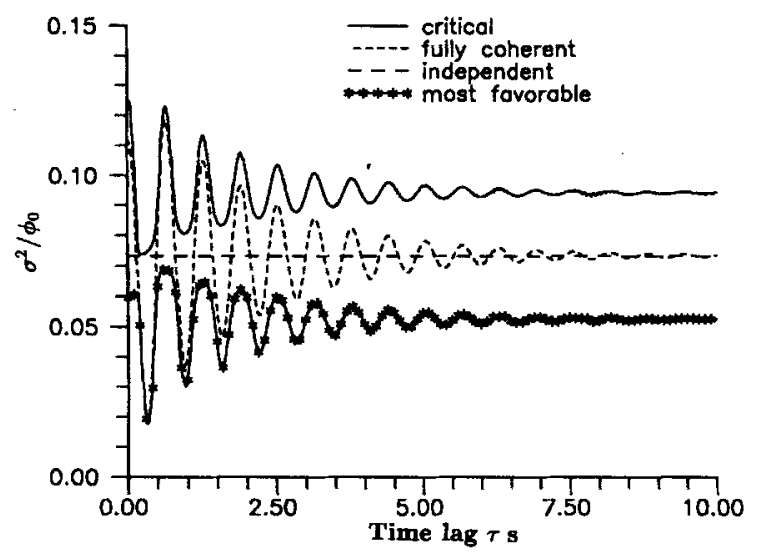

(b)

Figure 2. Response of SDOF system; $\omega_{0}=20.0 \mathrm{rad} / \mathrm{s} ; \eta=0.05:$ (a) PSD functions; $\tau=1 \mathrm{~s}$; (b) bounds on response variance

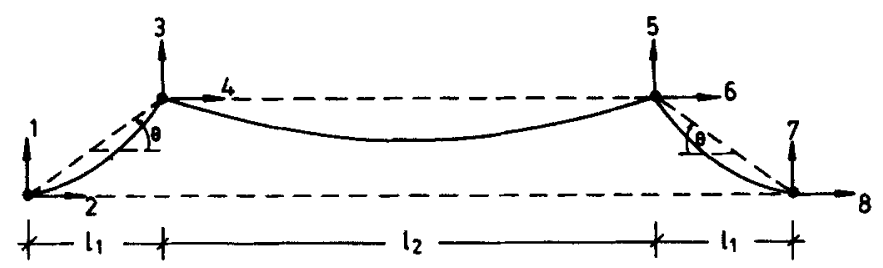

Figure 3. Three span suspended cable

matrices and then assembling them following the standard procedures of matrix methods of structural analysis. It may be recalled here that the dynamic stiffness coefficient $K_{i j}(\omega)$ is defined as the amplitude of a harmonic excitation of frequency $\omega$ to be applied at node $i$ to produce a unit harmonic displacement of the same frequency at the node $j$, with all other nodes being held fixed. These coefficients, apart from being frequency dependent, are complex in nature when damping or gyroscopic effects are present. The present authors have recently developed a space domain direct integration scheme for evaluating the dynamic stiffness coefficients of a sagged cable element, ${ }^{22}$ allowing for several complicating effects of cable dynamics such as inclined chord, axial translation, three dimensional coupling and proportional/non-proportional damping mechanisms. This scheme is particularly suited for systems with densely spaced modes, as is the case 
in the long span cable structure being studied here, for which the more popular finite element approach proves to be unwieldy because of very fine discretization needed to deal with the large number of participating modes.

Denoting the nodal force and displacement amplitude vectors, respectively, by $\mathbf{f}$ and $\delta$, which, for the structure shown in Figure 3, are of the size $8 \times 1$, the input-output relation in terms of the dynamic stiffness matrix $\mathbf{K}$ can be written as

$$
\mathbf{f}(\omega)=\mathbf{K}(\omega) \delta(\omega)
$$

Based on this expression, it is possible to carry out a stationary random response analysis and it can be shown that a typical output power spectral density function when the structure is acted upon by stationary excitations $x_{i}(t), i=1, \ldots, 8$, along the directions $i=1, \ldots, 8$, respectively, can be expressed in the form

$$
S_{u}(\omega)=\left\{\sum_{i=1}^{8} S_{i i}(\omega) H_{i i}(\omega)+\sum_{i \neq j=1}^{8}\left|S_{i j}\right| H_{i j}\left(\omega, \tau_{1}, \tau_{2}, \tau_{3}\right)\right\}\left|H_{\mathrm{f}}(\omega)\right|^{2}
$$

Here $\tau_{1}, \tau_{2}$ and $\tau_{3}$ are, respectively, the time lags in seconds occurring at the successive supports reckoned from the left with respect to the left hand support. Thus, for example, for the PSD of the force transmitted to the support along direction 3 , one gets

$$
\begin{gathered}
H_{11}=\left|K_{31}\right|^{2}, \quad H_{22}=\left|K_{32}\right|^{2}, \quad H_{33}=\left|K_{33}\right|^{2}, \quad H_{44}=\left|K_{34}\right|^{2}, \quad H_{55}=\left|K_{35}\right|^{2}, \quad H_{66}=\left|K_{36}\right|^{2} \\
H_{77}=\left|K_{37}\right|^{2}, \quad H_{38}=\left|K_{38}\right|^{2}, \quad H_{12}=2 \operatorname{Re}\left\{\bar{K}_{31} K_{32}\right\}, \quad H_{13}=2 \operatorname{Re}\left\{\bar{K}_{31} K_{33} \exp \left(-\mathrm{i} \omega \tau_{1}\right)\right\} \\
H_{14}=2 \operatorname{Re}\left\{\bar{K}_{31} K_{34} \exp \left(-\mathrm{i} \omega \tau_{1}\right)\right\}, \quad H_{15}=2 \operatorname{Re}\left\{\bar{K}_{31} K_{35} \exp \left(-\mathrm{i} \omega\left(\tau_{1}+\tau_{2}\right)\right)\right\} \\
H_{16}=2 \operatorname{Re}\left\{\bar{K}_{31} K_{36} \exp \left(-\mathrm{i} \omega\left(\tau_{1}+\tau_{2}\right)\right)\right\}, \quad H_{17}=2 \operatorname{Re}\left\{\bar{K}_{31} K_{37} \exp \left(-\mathrm{i} \omega\left(\tau_{1}+\tau_{2}+\tau_{3}\right)\right)\right\} \\
H_{18}=2 \operatorname{Re}\left\{\bar{K}_{31} K_{38} \exp \left(-\mathrm{i} \omega\left(\tau_{1}+\tau_{2}+\tau_{3}\right)\right)\right\}, \quad H_{23}=2 \operatorname{Re}\left\{\bar{K}_{32} K_{33} \exp \left(-\mathrm{i} \omega \tau_{1}\right)\right\} \\
H_{24}=2 \operatorname{Re}\left\{\bar{K}_{32} K_{34} \exp \left(-\mathrm{i} \omega \tau_{1}\right)\right\}, \quad H_{25}=2 \operatorname{Re}\left\{\bar{K}_{32} K_{35} \exp \left(-\mathrm{i} \omega\left(\tau_{1}+\tau_{2}\right)\right)\right\} \\
H_{26}=2 \operatorname{Re}\left\{\bar{K}_{32} K_{36} \exp \left(-\mathrm{i} \omega\left(\tau_{1}+\tau_{2}\right)\right)\right\}, \quad H_{27}=2 \operatorname{Re}\left\{\bar{K}_{32} K_{37} \exp \left(-\mathrm{i} \omega\left(\tau_{1}+\tau_{2}+\tau_{3}\right)\right)\right\} \\
H_{28}=2 \operatorname{Re}\left\{\bar{K}_{32} K_{38} \exp \left(-\mathrm{i} \omega\left(\tau_{1}+\tau_{2}+\tau_{3}\right)\right)\right\}, \quad H_{34}=2 \operatorname{Re}\left\{\bar{K}_{33} K_{34}\right\} \\
H_{35}=2 \operatorname{Re}\left\{\bar{K}_{33} K_{35} \exp \left(-\mathrm{i} \omega \tau_{2}\right)\right\}, \quad H_{36}=2 \operatorname{Re}\left\{\bar{K}_{33} K_{36} \exp \left(-\mathrm{i} \omega \tau_{2}\right)\right\} \\
H_{37}=2 \operatorname{Re}\left\{\bar{K}_{33} K_{37} \exp \left(-\mathrm{i} \omega\left(\tau_{1}+\tau_{2}\right)\right)\right\}, \quad H_{38}=2 \operatorname{Re}\left\{\bar{K}_{33} K_{38} \exp \left(-\mathrm{i} \omega\left(\tau_{1}+\tau_{2}\right)\right)\right\} \\
H_{45}=2 \operatorname{Re}\left\{\bar{K}_{34} K_{35} \exp \left(-\mathrm{i} \omega \tau_{2}\right)\right\}, \quad H_{46}=2 \operatorname{Re}\left\{\bar{K}_{34} K_{36} \exp \left(-\mathrm{i} \omega \tau_{2}\right)\right\} \\
H_{47}=2 \operatorname{Re}\left\{\bar{K}_{34} K_{37} \exp \left(-\mathrm{i} \omega\left(\tau_{1}+\tau_{2}\right)\right)\right\}, \quad H_{48}=2 \operatorname{Re}\left\{\bar{K}_{34} K_{38} \exp \left(-\mathrm{i} \omega\left(\tau_{1}+\tau_{2}\right)\right)\right\} \\
H_{56}=2 \operatorname{Re}\left\{\bar{K}_{35} K_{36}\right\}, \quad H_{57}=2 \operatorname{Re}\left\{\bar{K}_{35} K_{37} \exp \left(-\mathrm{i} \omega \tau_{1}\right)\right\}, \quad H_{58}=2 \operatorname{Re}\left\{\bar{K}_{35} K_{38} \exp \left(-\mathrm{i} \omega \tau_{1}\right)\right\} \\
H_{67}=2 \operatorname{Re}\left\{\bar{K}_{36} K_{37} \exp \left(-\mathrm{i} \omega \tau_{1}\right)\right\}, \quad H_{68}=2 \operatorname{Re}\left\{\bar{K}_{36} K_{38} \exp \left(-\mathrm{i} \omega \tau_{1}\right)\right\} \\
H_{78}=2 \operatorname{Re}\left\{\bar{K}_{37} K_{38}\right\}
\end{gathered}
$$

Here bars denote complex conjugation. Similarly, an expression for the PSD of the force transmitted to the support in direction 4 can be obtained from the above expression by replacing stiffness coefficients of the form $K_{3 i}$ by $K_{4 i}$.

The problem of critical excitation consists of finding the modulus of the CPSD functions $\left|S_{i j}(\omega)\right|, i \neq j$, which produce the highest response variance given by the area under the PSD function given above and subject to the constraints

$$
0 \leqslant\left|S_{i j}(\omega)\right| \leqslant \sqrt{S_{i i}(\omega) S_{j j}(\omega)}, \quad i, j=1, \ldots, 8, \quad i \neq j
$$


It may be emphasized here that equation (21) is a generalization of equation (5) and, therefore, the solution to the problem of finding the critical CPSD function follows the same logic as has been used in deriving equation (17). Accordingly, the modulus of the critical CPSD functions is obtained as

$$
\begin{gathered}
\left|S_{i j}(\omega)\right|=0 \text { if } H_{i j}\left(\omega, \tau_{1}, \tau_{2}, \tau_{3}\right)<0 \\
\left|S_{i j}(\omega)\right|=\sqrt{S_{i i}(\omega) S_{j j}(\omega)} \text { if } H_{i j}\left(\omega, \tau_{1}, \tau_{2}, \tau_{3}\right)>0, \quad i \neq j, i, j=1, \ldots, 8
\end{gathered}
$$

Conversely, the most favourable modulus of CPSD functions which produce the least response can also easily be obtained as

$$
\begin{gathered}
\left|S_{i j}^{*}(\omega)\right|=0 \quad \text { if } H_{i j}\left(\omega, \tau_{1}, \tau_{2}, \tau_{3}\right)>0 \\
\left|S_{i j}^{*}(\omega)\right|=\sqrt{S_{i i}(\omega) S_{j j}(\omega)} \quad \text { if } H_{i j}\left(\omega, \tau_{1}, \tau_{2}, \tau_{3}\right)<0, \quad i \neq j, i, j=1, \ldots, 8
\end{gathered}
$$

For the purpose of illustration, the response of the cable structure is analysed for four cases, namely,

(i) when all the excitations $\ddot{x}_{i}(t), i=1, \ldots, 8$, are uncorrelated,

(ii) when all the excitations $\ddot{x}_{i}(t), i=1, \ldots, 8$, are fully correlated,

(iii) when the excitations have critical coherence properties and

(iv) when the excitations have the most favourable coherence properties.

Furthermore, it may be noted that each of the supports in this problem is acted upon by two components of ground motions. If an assumption that the principal directions of the structure coincide with the principal directions of the excitations is made, then the cross correlations between components of excitation at the same point can be taken to be zero. Thus, for example, with this assumption, $\ddot{x}_{1}(t)$ and $\ddot{x}_{2}(t)$ become uncorrelated, while $\ddot{x}_{2}(t)$ and $\ddot{x}_{3}(t)$ still remain correlated. To investigate the effect of such assumptions on the response, the calculations listed above are repeated incorporating these assumptions. Tables I and II summarize the results obtained on the system response from which it may be concluded that of all the possible scenarios which are likely to yield the bounds on the response, the ones which actually bound the response are those which correspond to equations (24) and (25). Time lag effects in this example are observed to reduce the response and, also, the case of all excitation components being independent produces force responses higher than that produced by fully coherent excitations. The response PSD functions are shown in Figures 4 and 5, from which it can be observed that the critical response PSD envelopes the functions corresponding to the case of independent and fully correlated excitations which, consequently, results in highest response variance. The coupling between longitudinal and transverse modes of cable vibration is also evident from these figures with the longitudinal modes contributing to higher peaks in the response PSD function. A few of the moduli of the critical CPSD functions, which produce highest response, are plotted in

Table I. Steady state variance of force transmitted to the cable support along direction 3

\begin{tabular}{ccccc}
\hline No. & $\begin{array}{l}\text { Nature of } \\
\text { coherence }\end{array}$ & Time lag (s) & $\begin{array}{c}\sigma_{\mathrm{F}}^{2} / E_{0} \text { for } \\
\text { case A }\end{array}$ & $\begin{array}{c}\sigma_{\mathrm{F}}^{2} / E_{0} \\
\text { case B }\end{array}$ \\
\hline 1 & Critical & $\tau_{1}=0 \cdot 25, \tau_{2}=1.25, \tau_{3}=1.5$ & $0.019 \times 10^{11}$ & $0.018 \times 10^{11}$ \\
2 & Fully coherent & $\tau_{1}=0 \cdot 25, \tau_{2}=1 \cdot 25, \tau_{3}=1.5$ & $0.039 \times 10^{10}$ & $0.011 \times 10^{11}$ \\
3 & Incoherent & $\tau_{1}=0 \cdot 25, \tau_{2}=1 \cdot 25, \tau_{3}=1.5$ & $0.012 \times 10^{11}$ & $0.012 \times 10^{11}$ \\
4 & Most favourable & $\tau_{1}=0.25, \tau_{2}=1 \cdot 25, \tau_{3}=1.5$ & $0.021 \times 10^{10}$ & $0.024 \times 10^{10}$ \\
5 & Critical & $\tau_{1}=0, \tau_{2}=0, \tau_{3}=0$ & $0.023 \times 10^{11}$ & $0.022 \times 10^{11}$ \\
6 & Fully coherent & $\tau_{1}=0, \tau_{2}=0, \tau_{3}=0$ & $0.031 \times 10^{10}$ & $0.011 \times 10^{11}$ \\
7 & Incoherent & $\tau_{1}=0, \tau_{2}=0, \tau_{3}=0$ & $0.012 \times 10^{11}$ & $0.012 \times 10^{11}$ \\
8 & Most favourable & $\tau_{1}=0, \tau_{2}=0, \tau_{3}=0$ & $0.016 \times 10^{10}$ & $0.018 \times 10^{10}$ \\
\hline
\end{tabular}

Case A: excitation components at the same point are correlated; case B: excitation components at the same point are uncorrelated 
Table II. Steady state variance of force transmitted to the cable support along direction 4

\begin{tabular}{ccccc}
\hline No. & $\begin{array}{c}\text { Nature of } \\
\text { coherence }\end{array}$ & Time lag (s) & $\begin{array}{c}\sigma_{\mathrm{F}}^{2} / E_{\mathrm{O}} \text { for } \\
\text { case A }\end{array}$ & $\begin{array}{c}\sigma_{\mathrm{F}}^{2} / E_{0} \\
\text { case B }\end{array}$ \\
\hline 1 & Critical & $\tau_{1}=0.25, \tau_{2}=1.25, \tau_{3}=1.5$ & $0.081 \times 10^{11}$ & $0.072 \times 10^{11}$ \\
2 & Fully coherent & $\tau_{1}=0.25, \tau_{2}=1.25, \tau_{3}=1.5$ & $0.024 \times 10^{11}$ & $0.040 \times 10^{11}$ \\
3 & Incoherent & $\tau_{1}=0.25, \tau_{2}=1.25, \tau_{3}=1.5$ & $0.048 \times 10^{11}$ & $0.048 \times 10^{11}$ \\
4 & Most favourable & $\tau_{1}=0.25, \tau_{2}=1.25, \tau_{3}=1.5$ & $0.013 \times 10^{11}$ & $0.016 \times 10^{11}$ \\
5 & Critical & $\tau_{1}=0, \tau_{2}=0, \tau_{3}=0$ & $0.091 \times 10^{11}$ & $0.083 \times 10^{11}$ \\
6 & Fully coherent & $\tau_{1}=0, \tau_{2}=0, \tau_{3}=0$ & $0.022 \times 10^{11}$ & $0.038 \times 10^{11}$ \\
7 & Incoherent & $\tau_{1}=0, \tau_{2}=0, \tau_{3}=0$ & $0.048 \times 10^{11}$ & $0.048 \times 10^{11}$ \\
8 & Most favourable & $\tau_{1}=0, \tau_{2}=0, \tau_{3}=0$ & $0.010 \times 10^{11}$ & $0.012 \times 10^{11}$ \\
\hline
\end{tabular}

Case A: excitation components at the same point are correlated; case B: excitation components at the same point are uncorrelated

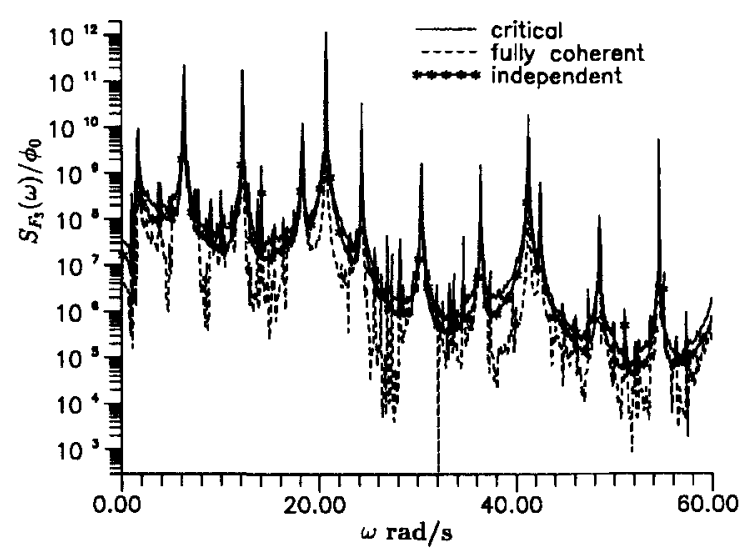

Figure 4. PSD functions of force transmitted to the cable support in direction 3

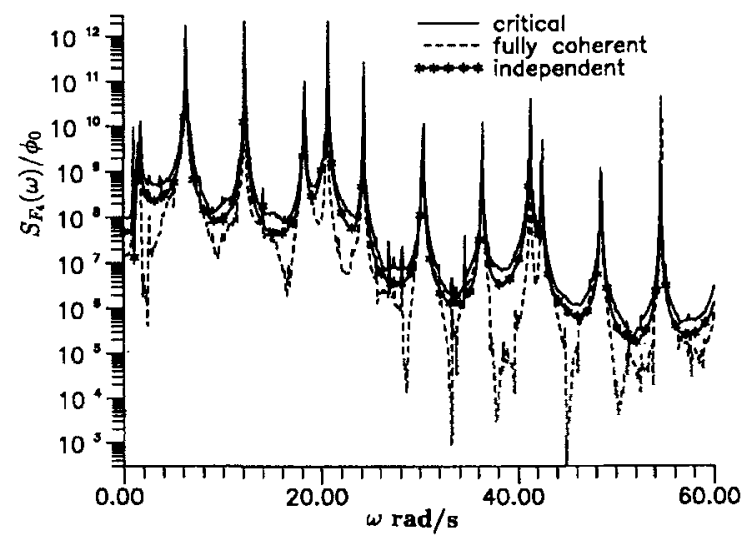

Figure 5. PSD functions of force transmitted to the cable support in direction 4

Figure 6, from which it can be shown that they consist of an alternating sequence of frequency windows in

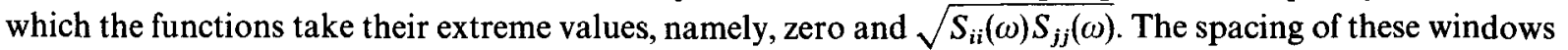
differs for each of the CPSD functions and is governed by the zeros of the associated transfer functions $H_{i j}\left(\omega, \tau_{1}, \tau_{2}, \tau_{3}\right)$. 

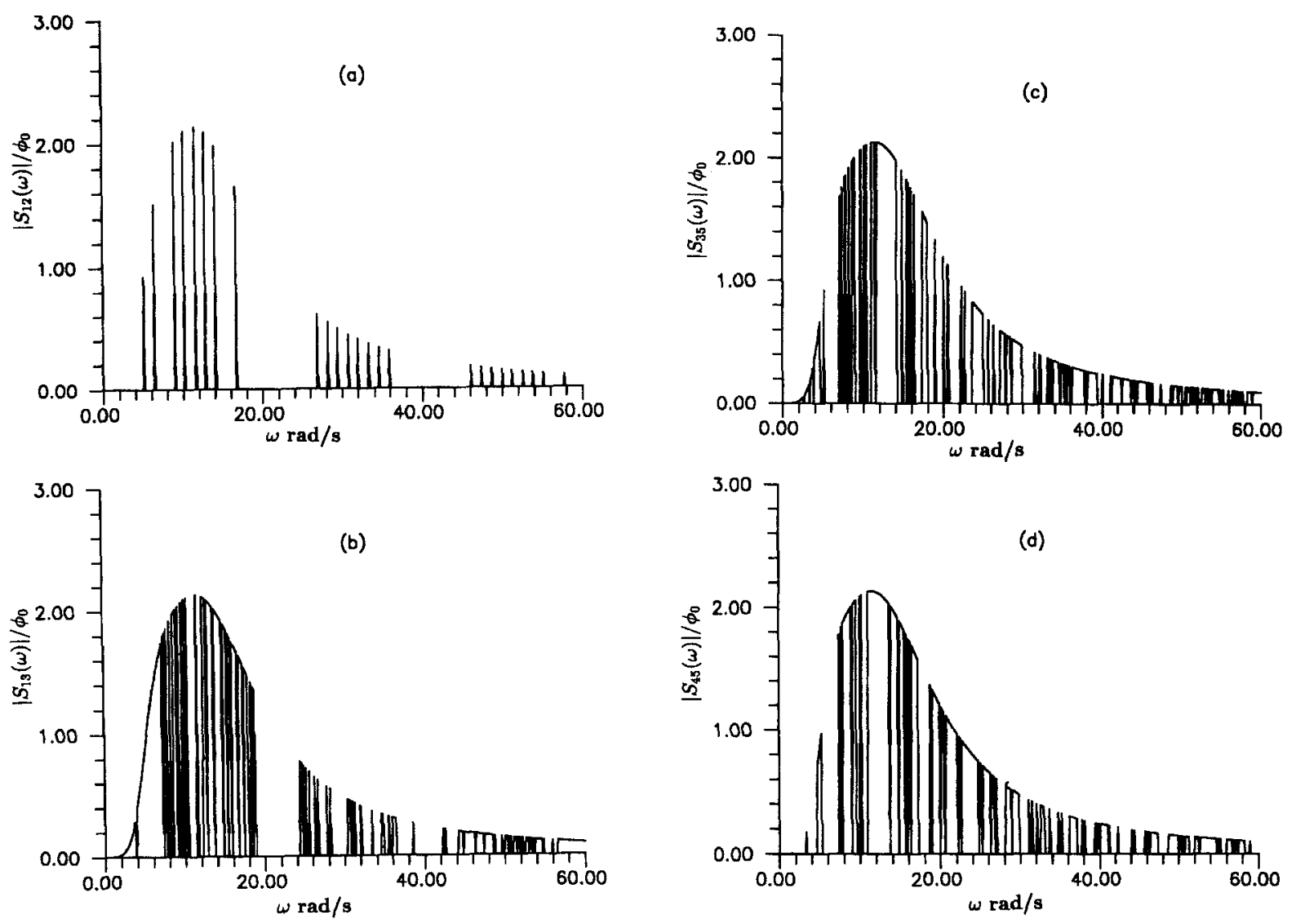

Figure 6. Modulus of the critical CPSD functions corresponding to the results of Figure 4

\section{PIPING SYSTEM}

Next, we consider the idealized piping structure shown in Figure 7 which has two supports, each of which carries a two dimensional support motion. The piping properties assumed are $E=2.1 \times 10^{11} \mathrm{~N} / \mathrm{m}^{2}$, mass density $=7900 \mathrm{~kg} / \mathrm{m}^{3}, A=7.068 \times 10^{-4} \mathrm{~m}^{2}$, second moment of area $=1.688 \times 10^{-6} \mathrm{~m}^{4}$ and damping coefficient $=2$ per cent for all the modes. The autospectral density functions of all the support motions are again taken to be as given by equation (19) with $\omega_{\mathrm{g}}=10 \pi \mathrm{rad} / \mathrm{s}$ and $\zeta_{\mathrm{g}}=0.6$. The time lag $\tau$ for this example is taken to be zero. This example serves to illustrate two points: firstly, this structure is typical of multisupported secondary systems which are found in industrial buildings and, secondly, we employ a readily available finite element structural analysis code (NISA) to derive the critical CPSD functions. For the purpose of illustration, the displacement $u_{x}$ in the $x$-direction at the point $(0,-4,-1)$ is taken as the response variable of interest. The PSD function of this variable can again be expressed in the form of equation (21). The summations here, however, run from $i=1$ to 4 . The steps employed in the calculation of the generalized transfer functions $H_{i j}(\omega), i, j=1, \ldots, 4$, are:

1. Select $i$ and $j$; release the degree of freedom along the $i$ th and $j$ th directions; add two large masses $M$ (about $10^{6}$ times the total mass of the structure) at the support along these two directions.

2. Perform the free vibration analysis of this modified structure.

3. Apply two independent white noise forces of intensities $M^{2}$ along the $i$ th and $j$ th directions, respectively, and perform random vibration analysis to compute the PSD of $u_{x}$, denote it by $S_{1}(\omega)$. Notice 
that if $i=j$, we would be applying only one excitation, and, for this case, $H_{i i}(\omega)=S_{1}(\omega) / M^{2}$; proceed to step 6.

4. Apply two fully correlated white noise forces of intensities $M^{2}$ along the $i$ th and $j$ th directions and perform random vibration analysis to compute the PSD of $u_{x}$ and denote it by $S_{2}(\omega)$.

5. Compute $H_{i j}(\omega)=\left[S_{2}(\omega)-S_{1}(\omega)\right] / M^{2}$.

6. Change $i$ and $j$ and repeat steps 1-5.

Once the generalized transfer functions $H_{i j}$ are thus found, the determination of the least favourable and the most favourable CPSD functions and the associated responses follow the same procedure as has been used for the cable structure, see equations (23) and (24). Figure 8 shows the PSD of the displacement $u_{x}$ produced by the critical excitation along with those produced by the most favourable, independent and fully correlated excitations. The response variances normalized with respect to the input variance $E_{0}$ produced by these four excitations were computed to be, respectively, $1.274 \times 10^{-1}, 0.30 \times 10^{-1}, 0.557 \times 10^{-1}$ and $0.907 \times 10^{-1}$. It may be noted that, for the input PSD given in equation (19), $E_{0}=\left(\pi \phi_{0} \omega_{\mathrm{g}} / 2 \zeta_{\mathrm{g}}\right)\left(1+4 \zeta_{\mathrm{g}}^{2}\right)$. Figure 9 shows the critical $\left|S_{i j}(\omega)\right|$ between components of excitations at the two supports which, again, consist of an alternating sequence of frequency windows in which the functions take their extreme permissible values, namely, zero and $\sqrt{S_{i i}(\omega) S_{j j}(\omega)}$.

\section{CONCLUSIONS}

A new method for determining the bounds on the response of multi-supported structures subjected to incompletely specified multi-component earthquake excitations is outlined. The excitation is modelled as a stationary vector Gaussian random process. The diagonal terms in the excitation power spectral density function matrix are taken to be known, while the off-diagonal terms, which represent the cross spectral density functions, are assumed to be not given. This type of situation is easily conceivable when the seismic inputs are specified through a set of response spectra which, by definition, do not consider the crosscorrelation effects between different excitations. While the autospectral density functions compatible with different response spectrum components can be generated using standard random vibration theory, the information on cross spectra is not easy to generate. Besides, models for spatial variability of seismic ground motions are less readily available than models for single point excitations. Thus, the approach outlined in this study, which leads to the determination of the most unfavourable cross spectral density functions, can provide useful information for assessing the safety of structures. Moreover, these bounds, being exact in nature, can be used for assessing the accuracy of alternative methods of multi-support seismic excitation problems. The critical CPSD functions are shown to be dependent on the system properties and also on the

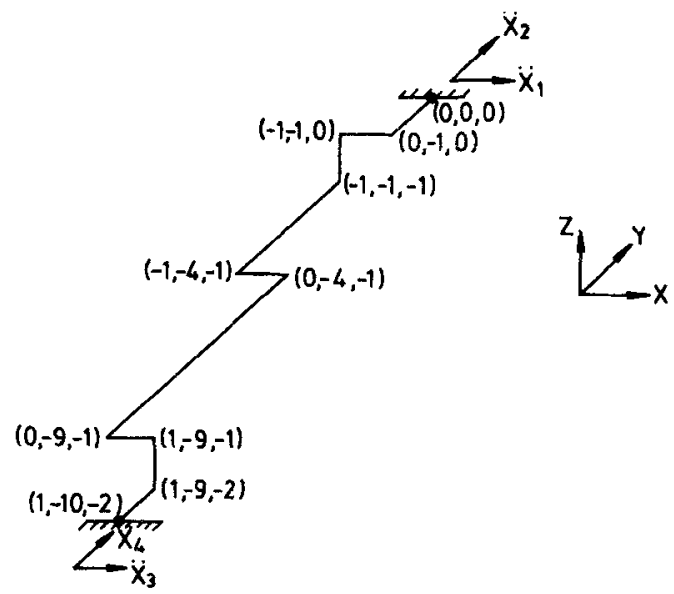

Figure 7. Idealized piping system; all co-ordinates are in $\mathrm{m}$ 


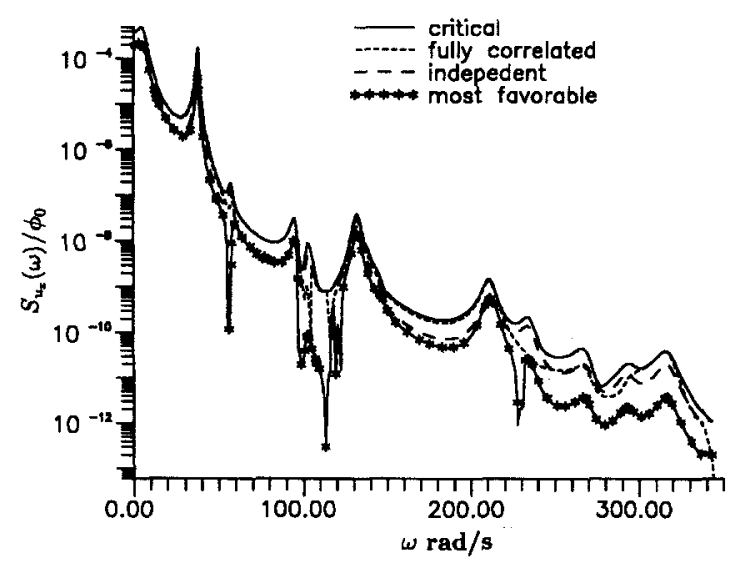

Figure 8. PSD functions of displacement $u_{x}$
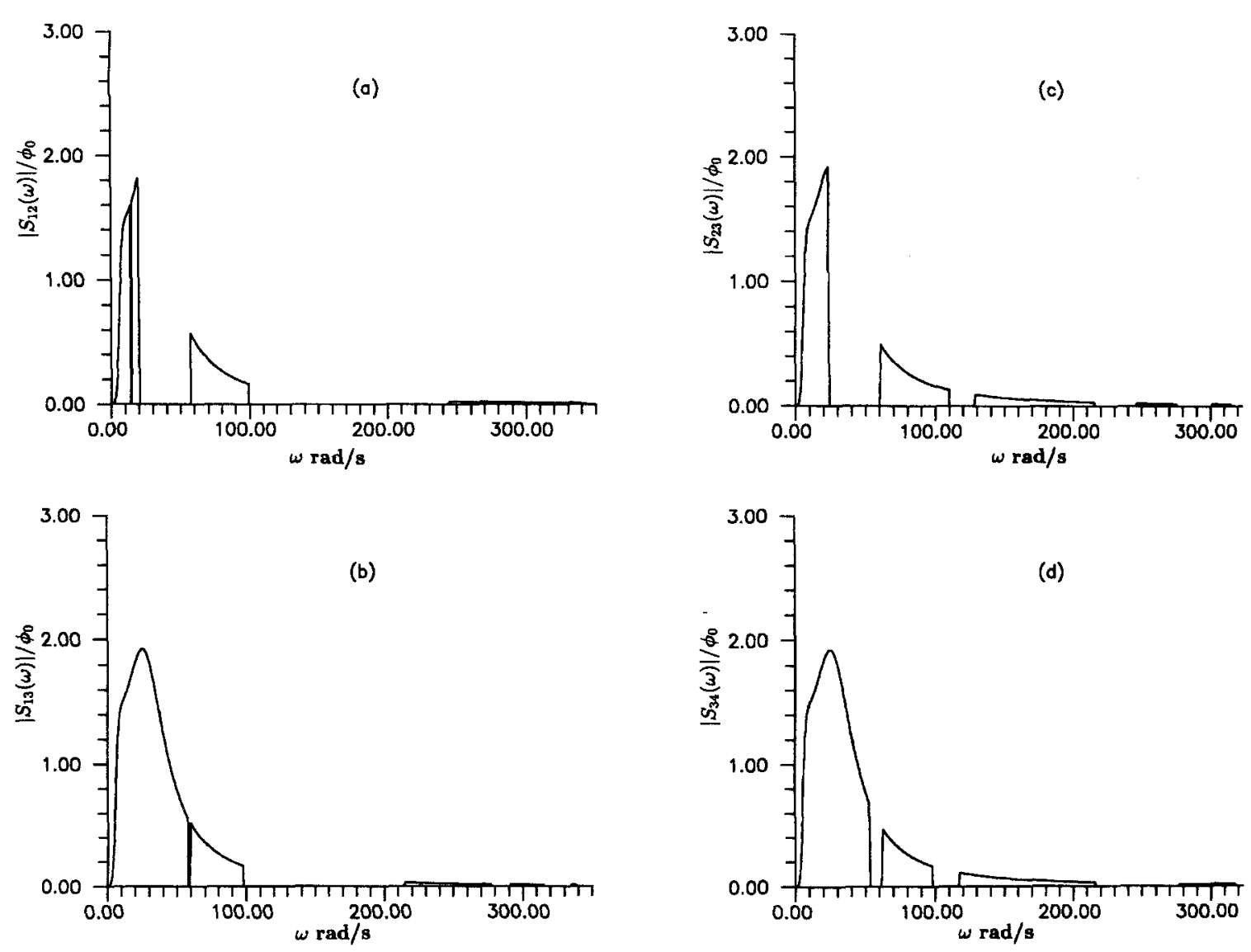

Figure 9. Modulus of the critical CPSD functions corresponding to the results of Figure 8

previously specified autospectral density functions. The derivation of these functions is based on the development of a set of generalized transfer functions associated with a given response variable. This paper demonstrates the determination of these functions for two typical multi-supported structures using the dynamic stiffness matrix method and the more popular finite element method. 
The response variable chosen for maximization in this study has been the total response. However, in practice, the response is often split into three components, namely, the dynamic component, the pseudo-static component and the cross component which reflects the effect of correlation between the dynamic and pseudo-static parts [see equation (10)]. In the design of multiply supported piping systems, the dynamic response component is taken to be primary response variable, with the other responses being treated as secondary. Furthermore, higher allowable stresses are specified against total response and against secondary stresses. ${ }^{18}$ The present study can easily be extended to incorporate different levels of importance for the three response components in determining the critical CPSD functions; further work, however, needs to be done to examine the relationship between response spectrum based methods and the results developed in this study.

\section{ACKNOWLEDGEMENTS}

This work was supported by funding from the Government of India Department of Science and Technology, which is gratefully acknowledged.

\section{REFERENCES}

1. N. C. Nigam and S. Narayanan, Applications of Random Vibrations, Narosa, New Delhi, 1994.

2. R. F. Drenick, 'Model-free design of aseismic structures', J. eng. mech. div. ASCE 96, 483-493 (1970).

3. R. F. Drenick, 'Aseismic design by way of critical excitations', J. eng. mech. div. ASCE 99, 649-667 (1973).

4. P. C. Wang and C. B. Yun, 'Site-dependent critical design spectra', Earthquake eng. struct. dyn. 7, $569-578$ (1979).

5. M. Srinivasan, B. Ellingwood and R. Corotis, 'Critical base excitations of structural systems', J. eng. mech. ASCE 117, 1403-1422 (1991).

6. R. N. Iyengar and C. S. Manohar, 'Nonstationary random critical seismic excitations', J. eng. mech. ASCE 113, 529-541 (1987).

7. C. S. Manohar and A. Sarkar, 'Critical earthquake input power spectral density function models for engineering structures', Earthquake eng. struct. dyn. 24, 1549-1566 (1995).

8. A. Sarkar and C. S. Manohar, 'Critical seismic vector random excitation for multiply supported structures', Probabilistic eng. mech. (under review).

9. A. Zerva, A. H. S. Ang and Y. K. Wen, 'Lifeline response to spatially variable ground motions', Earthquake eng. struct. dyn. 16, 361-379 (1988).

10. A. Zerva, 'Effect of spatial variability and propagation of seismic ground motions on the response of multiply supported structures', Probabilistic eng. mech. 6, 212-221 (1991).

11. H. Hao, 'Arch response to correlated multiple excitations', Earthquake eng. struct. dyn. 22, $389-404$ (1993).

12. R. S. Harichandran and W. Wang, 'Response of indeterminate two-span beam to spatially varying seismic excitation', Earthquake eng. struct. dyn. 19, 173-187 (1990).

13. P. Banerjee, 'Earthquake ground motion characterization for large or multiply supported structures', Current sci. 67, 386-394 (1994).

14. M. C. Lee and J. Penzien, 'Stochastic analysis of structures and piping systems subjected to stationary muitiple support excitations', Earthquake eng. struct. dyn. 11, 91-110 (1983).

15. R. A. Burdisso and M. P. Singh, 'Multiply supported secondary systems part I: response spectrum analysis', Earthquake eng. struct. dyn. 15, 53-72 (1987).

16. M. P. Singh and R. A. Burdisso, 'Multiply supported secondary systems part II: seismic inputs', Earthquake eng. struct. dyn. 15, 73-90 (1987).

17. T. Igusa and A. Der Kiureghian, 'Dynamic response of multiply supported secondary systems', J. eng. mech. ASCE 111, 20--41 (1985).

18. M. P. Singh, R. A. Burdisso and G. O. Maldonado, 'Methods used for calculating seismic response of multiply supported piping systems', J. pressure vessel technol. ASME 114, 46-52 (1992).

19. M.P. Singh and M. G. Ashtiany, 'Structural response under multicomponent earthquakes', J. eng. mech. ASCE 110, 761-775 (1984).

20. R. H. Clough and J. Penzien, Dynamics of Structures, 2nd edn, McGraw-Hill Kogakusha, New York, 1992.

21. M. Paz, Structural Dynamics, CBS Publishers, New Delhi, 1984.

22. A. Sarkar and C. S. Manohar, 'Dynamic stiffness matrix of a general cable element by numerical integrations in spatial domain', Arch. appl. mech. (accepted). 